\title{
Internacionalización, formación y análisis de la realidad. Tres conceptos esenciales en el desarrollo de acciones positivas por parte de FEDER (Federación Española de Enfermedades Raras)
}

\author{
Internationalization, training and analysis \\ of reality. Three essential concepts in the \\ development of positive actions by FEDER
}

Palabras Clave

FEDER, enfermedades raras, internacional, formación.

\section{Keywords}

FEDER, rare diseases, international, training.

\section{Presentación}

Las enfermedades raras (en adelante ER) o poco frecuentes (EPF) son aquellas que afectan a menos de 5 por cada 10.000 personas. Son enfermedades crónicas, generalmente graves y en su mayor parte de origen genético. Hay identificadas unas 7.000. Se estima que puede haber 3.000 .000 de personas afectadas por alguna de ellas en nuestro país.

A pesar del avance obtenido en los últimos años, las ER siguen constituyendo un tema 'emergente' para la sociedad en general (Aymé y Schmidtke, 2007:I477). Pero, al mismo tiempo, puede que estemos hablando no sólo de una novedad temática, sino también, como hace Francesc Palau (20I0:I6I), de un "nuevo paradigma en la medicina clínica”. Las ER necesitan un enfoque sociopoblacional, distinto al esencialista o al nominalista y que implica valoraciones compartidas, miradas holísticas, la incorporación de experiencia socio-cultural complementaria a la genética, la

\section{Antonio M. Bañón Hernández} <amhernan@ual.es>

Observatorio de las Enfermedades

Raras de FEDER.

Centro de Investigación en

Comunicación y Sociedad de la

Universidad de Almería

\section{Javier Fornieles Alcaraz} <jforniel@ual.es>

Observatorio de las Enfermedades Raras de FEDER.

Centro de Investigación en Comunicación y Sociedad de la Universidad de Almería
Para citar:

Bañón Hernández, A.M. y

Fornieles Alcaraz, J. (20I3):

"Internacionalización, formación y análisis de la realidad. Tres conceptos esenciales en el desarrollo de acciones positivas por parte de FEDER (Federación Española de Enfermedades Raras)". Revista Española de Discapacidad, I (2): I35-I4I.

$<$ http://dx.doi.org/IO.5569/23405IO4.01.02.08> 
disposición de aproximaciones multidisciplinares, preventivas (en sentido amplio) e integradoras, y medidas educativas (Palau, 20I0:I64). Estas patologías también suponen, por cierto, una oportunidad para la investigación (Griggs, 2006; Schieppati et al., 2009).

En los últimos I 5 años, se ha ido conformando en España una red de trabajo y atención a las personas con EPF en la que el movimiento asociativo ha tenido un alto protagonismo, siguiendo el proceso de conversión del enfermo paciente en protagonista justamente (Castillo, 2009). La Federación Española de Enfermedades Raras (FEDER) y sus más de 240 asociaciones han sido algunas de esas protagonistas. Con el paso de los años, la Federación ha ido fortaleciendo su modelo de trabajo apostando por acciones e intervenciones que estuviesen sustentadas en conceptos tales como la internacionalización, la formación constante o el análisis de la realidad. Las EPF constituyen un reto para todos (García-Ribes, 2006) y requiere modelos de trabajo que lo asuman, también por parte del movimiento asociativo. Este 20I3, Año Nacional de las Enfermedades Raras, FEDER ha seguido apostando por esos conceptos, como veremos a lo largo de estas páginas.

2. Internacionalización. EI Primer Encuentro Iberoamericano de Enfermedades Raras

Un objetivo fundamental para FEDER ha sido siempre la participación en redes internacionales de colaboración en el ámbito de las EPF, y también su promoción. A la intensa participación que, durante todos estos años, la Federación ha tenido en EURORDIS (Rare Disease Europe), hay que sumar ahora, en efecto, el papel promotor que ha asumido para generar una plataforma iberoamericana de asociaciones y federaciones de asociaciones vinculadas a las EPF.

Entre los días I4 y I 8 de octubre de 20I3, se celebró en Totana (Murcia) el Primer Encuentro
Iberoamericano de Enfermedades Raras, dirigido a profesionales y a representantes de asociaciones, y organizado por la Asociación D’Genes y por la Federación Española de Enfermedades Raras. Participaron los siguientes colectivos: Federación Colombiana de Enfermedades Raras (FECOER), Federación Argentina de Enfermedades Poco Frecuentes (FADEPOF), Asociación Todos Unidos Enfermedades Raras de Uruguay (ATUERU), Mundo Marfan Latino, Fundación CRONICARE de Colombia, Fundación Ecuatoriana de Pacientes con Enfermedades de Depósito Lisosomal (FEPEL-DASHA), Federación Mexicana de Enfermedades Raras (FEMEXER), Grupo Argentino de Ayuda al Paciente con Enfermedades Raras (GADAPER), Asociación Colombiana de Pacientes con Enfermedades de Depósito Lisosomal (ACOPEL), Organización Mexicana de Enfermedades Raras (OMER), Asociación Paolista de Mucopolisacaridosis y Enfermedades Raras (AMPS e Rarissimas) de Brasil, Federación de Enfermedades Raras de Portugal (FEDRA), Servicio de Información y Orientación de Guatemala, y Fundación Augusto Turenne de Uruguay. El impacto geográfico del encuentro fue, como puede apreciarse, muy alto. Y puede imaginarse, de igual manera, el gran trabajo de gestión que este evento supuso para D'Genes y para FEDER.

Sin duda, el desarrollo de un movimiento internacional es determinante para conseguir mejoras sólidas y duraderas en la situación diagnóstica, en la búsqueda de tratamientos y en el desarrollo de medidas que logren hacer más equitativo el acceso a recursos socio-educativos o psico-educativos, y que permitan, igualmente, promover mensajes menos discriminatorios y más positivos con respecto a las personas con EPF y sus familiares a través de los medios de comunicación. Y en ese movimiento internacional hay que ir haciendo redes cada vez más amplias. El primer encuentro no sirvió sólo para ampliar los vínculos de FEDER y FEDRA de los países iberoamericanos anteriormente mencionados, sino también para ampliar las conexiones entre éstos y EURORDIS. La Plataforma Europea, en efecto, estuvo muy 
presente durante los cuatro días; de hecho, su director, Yann Le Cam, participó el primer día del encuentro; y más tarde también lo hicieron John Dart (hablando del modelo asistencial europeo para las ER), Anja Helm (exponiendo las líneas fundamentales del Día Internacional de las ER 20I4) o Denis Costello (presentando la Red Social 'Rare Connect').

De estos eventos, han de salir conclusiones y decisiones. Es decir, la palabra ha de ir acompañada por la acción. Una de las consecuencias más importantes fue, naturalmente, la firma por parte de todos los asistentes de la Alianza Iberoamericana de Enfermedades Raras (ALIBER) y la constitución de su primera Junta Directiva. Pero además también se generó un clima de cooperación que habrá de ser el que marque el futuro trabajo de todos los colectivos implicados. Es importante conseguir proyectos conjuntos entre España e Iberoamérica y es esencial que los modelos de trabajo positivos se conozcan para que puedan exportarse de unos países a otros. De igual manera, hemos de conocer las experiencias negativas, para buscar su eliminación y la oferta de propuestas alternativas.

Este trabajo conjunto tendrá que cubrir las dimensiones político-institucionales, por un lado, pero también las científicas y las relacionadas con la dinámica propia del trabajo de las asociaciones. FEDER tiene un largo recorrido que puso en esos días, y seguirá poniendo, al servicio de las otras asociaciones y federaciones. Con la finalidad de informar justamente de este recorrido, en el ámbito de la orientación, de la promoción asociativa, de la intervención psicológica y educativa, de la comunicación y de la captación de fondos, y de la relación entre discapacidad y EPF, intervinieron distintas personas vinculadas a la Federación Española y a su Observatorio (OBSER): Claudia Delgado, Rosa Sánchez de Vega, Isabel Motero, Alba Ancochea, Justo Herranz, María Tomé, Estrella Mayoral, Luján Echandi, Tomás Castillo y Antonio M. Bañón.

Otro de los objetivos del encuentro era mostrar la importancia que en el caso español ha tenido el trabajo cooperativo entre distintos actores con responsabilidad en la gestión sociosanitaria, política o científica de las EPF. Así, por ejemplo, hay que destacar la presencia durante estos cuatro días de Manuel Posada, director del Instituto de Enfermedades Raras del Instituto de Salud Carlos III (IIER), de Francesc Palau, director científico del Centro de Investigación Biomédica en Red (CIBERER) y de Aitor Aparicio, director del Centro de Referencia Estatal de Atención a Personas con Enfermedades Raras y sus Familias (CREER). Así como las intervenciones de Pedro Serrano (presentando las conclusiones del proyecto europeo Social Economic Burden and Health-related Quality of Life in Patients with Rare Diseases in Europe, BURQOL-RD) y de María Luisa Martínez Frías (explicando la experiencia del Registro Español sobre Anomalías Congénitas, ECEMC). Cuando los movimientos asociativos están buscando establecer prioridades en el ámbito de las ER, conocer la calidad de vida de los afectados y generar registros de pacientes de confianza son dos excelentes puntos de partida, sin duda, ya que, en buena medida, esas informaciones servirán para argumentar mejor en el proceso reivindicativo de las asociaciones.

El éxito (siempre relativo y siempre mejorable, claro está) de este tipo de trabajo cooperativo ha de servir al resto de asociaciones de países que puedan estar en un punto más inicial del proceso de visibilización de las EPF y de la consecución de progresos legislativos, sanitarios y sociales; siempre partiendo del hecho de que las estructuras sanitarias son muy diversas y una misma solución o un mismo modelo de trabajo y colaboración no siempre es viable en todos los contextos geográficos. En cualquier caso, FEDER y, en general, el movimiento asociativo español en ER también aprendió mucho de las personas y de los colectivos que acudieron a Totana. Aprendió de la existencia de nuevos modelos de centros de referencia para la atención a las personas con EPF, del calado que pueden tener nuevas denominaciones 'oficiales' para estas patologías ('enfermedades catastróficas', por ejemplo), de la distancia que sigue habiendo en muchos países entre el decir 
y el hacer cuando se habla de compromiso político para los afectados y sus familias, de las consecuencias derivadas de la inexistencia de planes y registros o de la importancia de trabajar al lado de los medios de comunicación siendo participativos y originales al establecer ese vínculo.

Otro elemento trascendental en el caso español ha sido el compromiso que con la causa de las enfermedades poco frecuentes ha habido por parte de personas con gran transcendencia social e institucional. La vinculación de altavoces y portavoces a esta causa ha supuesto, sin duda, la consolidación de las EPF como un tema de interés social y sanitario (Bañón y Fornieles, 20II). En este sentido, hay que destacar la presencia en el encuentro de S.A.R. la Princesa de Asturias, quien, además, recibió y saludó personalmente a todos los participantes llegados de Europa y de Iberoamérica. Este es otro factor importante del modelo español de visibilización de las ER del que tomaron buena nota algunos de los invitados de D'Genes y de FEDER. Otros ya habían compartido experiencias similares. Los representantes de Portugal, Colombia, Argentina, Uruguay, México, Guatemala, Brasil, Venezuela, Ecuador y Argentina asistieron, ya finalizado el Primer Encuentro Iberoamericano, al VI Congreso Nacional de Enfermedades Raras (celebrado a partir del viernes I 8 de octubre y hasta el 20). La combinación del encuentro y del congreso tenía entre sus funciones la de ofrecer también a los representantes de algunos de estos países un modelo de trabajo con respecto a la organización de grandes eventos relacionados con las enfermedades poco frecuentes.

\section{Formación. Escuela de Formación CREER-FEDER: Formando líderes, inspirando acciones}

Las asociaciones de pacientes y en general los movimientos sociales no siempre perciben la importancia de la formación continua de sus trabajadores. Y tampoco perciben, en ocasiones, lo útil que es para el crecimiento del colectivo el intercambio de experiencias entre esos trabajadores y quienes constituyen, como protagonistas principales, ese movimiento asociativo: las personas con EPF, en nuestro caso. Es la mejor inversión posible, sin duda. La proyección internacional no puede dejar de lado que la fuerza de FEDER reside en sus asociaciones y en sus trabajadores, y que su formación debe ser una prioridad, como lo es el establecimiento de foros de intercambio de experiencias entre unas y otros. La cohesión de iniciativas y de mensajes también depende de este tipo de espacios compartidos. En todo caso, el trato directo siempre tiene un valor humano añadido para todos y facilita mejores intercambios comunicativos para el futuro, no sólo entre asociados y trabajadores, sino también entre los propios trabajadores o entre los mismos representantes de asociaciones.

Desde hace cuatro años, FEDER está desarrollando, junto con el Centro de Referencia Estatal de Atención a Personas con Enfermedades Raras y sus Familias (CREER), una experiencia denominada 'Escuela de Formación', orientada a cubrir, en parte, esa necesidad. Este año tuvo lugar la IV Edición, en Burgos. Sucedió el 20 y el 2I de septiembre y llevó por título 'Formando líderes, inspirando acciones'.

Son muchas las cuestiones que merecerían la pena ser abordadas en una escuela de formación, pero, por lo normal, hay que seleccionar contenidos que puedan ser tratados con profundidad en dos días de trabajo. Este año 2013 se compartieron experiencias sobre los siguientes temas: a) cómo deben mejorar los portavoces de las asociaciones con ER a la hora de expresar públicamente sus opiniones y sus reivindicaciones, especialmente si se hace a través de los medios de comunicación; b) experiencias innovadoras en la protección de datos en EPF; c) asistencia sanitaria a nivel europeo para las personas con ER; d) la Conferencia Europlan 20I4; d) la Red social Rare Connect; e) el Estudio Burqol-RD; f) la inclusión de los menores con ER en el entorno educativo; g) los Servicios de Información y Orientación; h) 
Formación online; y h) El Observatorio de las Enfermedades Raras (OBSER), una iniciativa para potenciar el conocimiento y el análisis de la información en ER.

\section{Análisis de la realidad: El Observatorio de las Enfermedades Raras de FEDER (OBSER)}

Uno de los problemas que, en nuestra opinión, sigue habiendo es el de la vinculación directa de las universidades españolas con las EPF. Para la observación e investigación de la realidad y para la identificación de buenas y malas prácticas, así como para la propuesta de pautas y esquemas de consolidación o de cambio de esas prácticas, los grupos de investigación universitarios pueden ser una herramienta sumamente útil para el movimiento asociativo de las ER. No en vano, a esos grupos universitarios se les pide cada vez más que desarrollen un trabajo de transferencia de sus investigaciones. En realidad, la transferencia tiene, naturalmente, una doble dirección. Lo ideal, en efecto, es que la universidad proyecte su trabajo hacia la sociedad pero, a la inversa, la sociedad tiene que hacer saber a la universidad por dónde van sus prioridades.

La Federación Española de Enfermedades Raras ha dado un importante paso en este sentido, vinculando el Observatorio de las Enfermedades Raras (OBSER) al Centro de Investigación en Comunicación y Sociedad (CySOC) de la Universidad de Almería. El OBSER servirá para identificar buenos y malos modelos en los siguientes ámbitos preferentemente: educativo, socio-sanitario, político y de los medios de comunicación.

Podemos decir que, en la actualidad, las universidades españolas apenas han contemplado como objeto de investigación las enfermedades raras en su conjunto; menos aún desde el punto de vista psicosocial o comunicativo. Salvo excepciones. Y esas excepciones con frecuencia han tenido que ver con la vinculación directa y personal de alguno de los investigadores con las enfermedades poco frecuentes. Esto hace, además, que la investigación social en enfermedades raras esté focalizada, por el momento, en determinadas universidades: Carlos III de Madrid, Cardenal Herrera, Oberta de Cataluña o Almería, por poner los ejemplos de centros más activos. Afortunadamente, al menos por el momento, los evaluadores de proyectos $\mathrm{I}+\mathrm{D}$ de los últimos años en los distintos ministerios implicados han apoyado las propuestas recibidas. Pero los avances que, aunque localizados, se han venido produciendo en estos años sólo se consolidarán si la inversión se mantiene. Y si otras entidades públicas y privadas apuestan por este campo de investigación.

Este año, el OBSER centrará sus análisis e investigaciones en tres ámbitos: el de los medios de comunicación, el educativo y el de política social y asociacionismo. Por lo que respecta al primero, en diciembre se presentarán los resultados del informe titulado El tratamiento de las enfermedades raras en los medios de comunicación españoles. 2012-2013. Seguiremos, en buena medida, el modelo de estudio cualitativo y cuantitativo, utilizado en un informe anterior sobre este mismo tema (Bañón et al., 20I I). En este informe, estarán implicadas las universidades de Almería, Valencia, Cardenal Herrera, Murcia y UOC. Además, contaremos con el apoyo y asesoramiento del Departamento de Comunicación de FEDER (coordinado por María Tomé). Con respecto a la educación, queremos hacer un estado de la cuestión sobre el tema que llevará por título La respuesta educativa a las necesidades de los alumnos con enfermedades poco frecuentes y sus familias. Será de gran ayuda el trabajo ya desarrollado en la Fundación FEDER (por ejemplo, Vasermanas y Frega, 2012) y el denominado 'Programa educativo de FEDER' (coordinado por Alba Ancochea). Y por lo que afecta a política social y asociacionismo, el OBSER se implicará en el estudio El asociacionismo relacionado con EPF en España. Historia y situación actual. Se prestará también especial atención a cómo las políticas sociosanitarias han afectado a la 
evolución o a la involución del movimiento asociativo. Será fundamental el apoyo del Departamento de Gestión Asociativa de FEDER (coordinado por Luján Echandi).

Por otro lado, además de la presencia en congresos nacionales e internacionales para difundir los resultados de los trabajos anteriormente mencionados, el OBSER iniciará la andadura de lo que ha llamado OBSER TV. En la página de internet del observatorio habrá una zona reservada a la presentación sistemática de vídeos en español en los que afectados, familiares, profesionales sociosanitarios, representantes de asociaciones, etc. hablen sobre las EPF. Seguimos aquí la idea desarrollada ya en EURORDIS TV.

Para el año 20I4, los responsables del Observatorio han propuesto la conversión del Informe Anual sobre Medios en estudios de seguimiento cuatrimestral de prensa, radio, televisión y redes sociales. Además, aprovechando la experiencia del documental El laberinto de las enfermedades raras, se utilizará el material audiovisual recopilado para la edición de dos nuevos documentales en los que el diagnóstico (o la ausencia del mismo) ocupará un papel central. No podemos olvidar que sigue vigente la recomendación que en el Estudio sobre situación de necesidades sociosanitarias de las personas con ER en España (Huete, A. y Díaz, E. 2009: 58 ) se hacía con respecto a la necesidad de generar iniciativas de atención a la problemática de los pacientes aislados. El asesoramiento del Servicio de Información y Orientación de FEDER (coordinado por Estrella Mayoral) permitirá identificar problemas esenciales narrados por los propios afectados. Esos problemas serán el punto de partida de la documentación para el desarrollo de estos documentales.

Igualmente, el OBSER participará en la organización del I Congreso Escolar Internacional de Enfermedades Raras. Es el primero de este tipo que se realiza en Europa. Es importante pensar en la innovación de propuestas en el área de las EPF. Se celebrará en La Salle Virgen del Mar de Almería. Por sus características, las ER nos introducen en un campo muy amplio de actividades profesionales y de cuestiones relacionadas con las ciencias sociales, la comunicación, la biología o la química. Los objetivos del congreso son los siguientes: a) Promover la realización de trabajos de calidad vinculados con estas ramas del conocimiento; b) Generar temas de reflexión interdisciplinares en el ámbito educativo; c) Conocer ejemplos y suscitar actitudes de superación ante problemas que surgen en la vida; d) Acercar las cuestiones y los conocimientos que se desarrollan en el aula a los actividades profesionales y las experiencias de la vida cotidiana; e) Realizar prácticas reales de comunicación escrita y oral mediante la exposición de resultados en el Congreso; f) Difundir, en este año de las ER, el conocimiento que existe de las mismas y el desafío científico y social que suponen y en el que todos podemos estar interesados e implicados; g) Fomentar el acercamiento y las relaciones interpersonales entre diversos centros y alumnos; y h) Conocer la labor y el importante papel que desarrollan las asociaciones de enfermedades poco frecuentes.

Más concretamente, las actividades previstas para el congreso son: I) Periódico o revista sobre enfermedades minoritarias; 2) Presentaciones orales con apoyo de Prezzi sobre enfermedades minoritarias y educación, su presencia en los medios, terapias y biotecnología; 3 ) Cómic relacionado con las enfermedades raras; 4) Documentos audiovisuales, 5) Blogs informativos, 6) Entrevista con responsables de la gestión sanitaria o con científicos; 7) Graffiti o mural desarrollado por alumnos de Bachillerato artístico, 8) Póster sobre algún aspecto vinculado al diagnóstico, tratamiento o atención socio-sanitaria de las ER, y 9) Actividades artísticas diversas.

En definitiva, las tres iniciativas descritas anteriormente van a permitir consolidar las líneas de trabajo de FEDER basadas en la internacionalización, en la formación continua y en el análisis de la realidad aprovechando la infraestructura de investigación universitaria. 


\section{Referencias bibliográficas}

Aymé, S. y Schmidtke, J. (2007): "Networking for rare diseases: a necessity for Europe". Bundesgesundheitsbl-GesundheitsforschGesundheitsschutz, I 2: I477-I483.

Bañón, A.M. et al. (eds.) (20II): Communication Strategies and Challenges for Rare Diseases: Medical Research as a Referent. A Quantitative-discursive Study of Spanish Printed and Digital Written Press (2009-2010). Valencia: CIBERER (Centre for Biomedical Network Research on Rare Diseases).

Bañón, A.M. y Fornieles, J. (2OII): "Communication and Rare Diseases: Contextualisation”, en A.M. Bañón et al. (eds.), Communication Strategies and Challenges for Rare Diseases: Medical Research as a Referent. A Quantitative-discursive Study of Spanish printed and digital written press. Valencia: CIBERER (Centro de Investigación Biomédica en Red para las Enfermedades Raras): I I-40.

Castillo, T. (2009): Aprendiendo a vivir. La enfermedad: descubrir las posibilidades que hay en mí. Barcelona: Ediciones CEAC.
Huete, A. y Díaz, E. (2009): Estudio sobre la situación de Necesidades Sociosanitarias de las personas con Enfermedades Raras en España. Madrid: Caja Madrid.

García-Ribes, M. et al. (2006): "Nuevos retos: el médico de familia ante las 'enfermedades raras'”. Atención Primaria, 37 (7): 369-370.

Griggs, R. et al. (2009): "Clinical research for rare disease: Opportunities, challenges, and solutions". Molecular Genetics and Metabolism, 96: 20-26.

Palau, F. (20IO): "Enfermedades raras, un paradigma emergente en la medicina del siglo XXI”. Medicina Clínica, I34 (4): I6I-I 68.

Schieppati, A. et al. (2008): "Why rare diseases are an important medical and social issue". The Lancet, 37I: 2039-204I.

Vasermanas, D. y Frega, Ma .A. (2012): Programa 'Yo también quiero estudiar'. Madrid: Fundación FEDER. 